\title{
Total Score
}

National Cancer Institute

\section{Source}

National Cancer Institute. Total Score. NCI Thesaurus. Code C120457.

The sum of a set of numeric values. 